\title{
Design and Implementation of Phase Difference Detection Device for Marine Power Station Based on S7-1200 PLC
}

\author{
Jia $\mathrm{Li}^{*}$ and Zhiliang $\mathrm{Wu}$ \\ ${ }^{1}$ College of Marine Electrical Engineering, Dalian Maritime University, Dalian 116026, China \\ ${ }^{*}$ Corresponding author
}

\begin{abstract}
Based on the analysis of the principle of phase difference detection for marine power station's parallel operation set, a method for improving the accuracy of phase difference detection is presented. This method uses the interruption mode to reset and read the current count value of high speed counter to measure lead time and accurately calculate the time point of giving a sign of switching on. On the experimental platform of physical simulation system of marine power station, the phase difference detection device of marine power station is designed by choosing S7-1200 PLC as the core controller. Complete the design of peripheral hardware circuit, as well as PLC configuration and programming. The accuracy and reliability of phase difference detection are verified, after many experiments on the physical simulation experimental platform of marine power station.
\end{abstract}

Keywords-marine power station; phase difference detection; 87 1200 plc; constant lead time; interruption

\section{INTRODUCTION}

Marine power station is responsible for providing safe, reliable and high quality power to the whole ship. So it is one of the most important parts of modern ships. In order to meet the requirements of reliability and economy of ship power station, SSC "Classification Rules of Steel Sea-going Vessel" requires each ship is equipped with at least two main generators In emergency conditions or heavy load conditions, generators must be powered by busbar to the ship in order to meet the requirements of different conditions[1].So, parallel operation becomes essential function of the marine power station. Compared with the traditional analog automatic parallel operation device, the digital automatic parallel operation device is more and more widely used with the improvement of the automation degree of the ship. And the superiority of its high accuracy is showed more and more obvious.

In this paper, phase difference detection device, as an important part of automatic parallel operation device, is designed. Its digital core controller is PLC S7-1200, whose functions of high speed counter and pulse width modulation output are fully utilized. The time point of qualified phase difference is accurately captured.

\section{NON-SYNCHRONOUS}

The voltage, generated by the generator at any time, can be expressed as:

$$
\dot{U}=A \sin (\omega t+\varphi)
$$

The voltage, at any time, is related to three basic parameters, such as amplitude A, angular frequency $\omega$ and phase angle $\varphi$. The ideal condition of parallel operation is that three basic parameters of standby generation's voltage is same with grid voltage. At this time, switch on main switch of standby generator, the circular current between both generators is zero. In fact, it is almost impossible to fully achieve the ideal condition. However, as long as the deviation of the three parameters is less, and the circular current within the limits of the system, it can ensure paralleling successfully.

Usually, frequency of marine power system is $50 \mathrm{~Hz}$ or $60 \mathrm{~Hz}$. In $20 \mathrm{~ms}$ or even shorter time, the phase changes from 0 degrees to 360 degrees. So, it is difficult but important for designing parallel controller to detect phase difference exactly in real-time.

\section{Principle of Phase DifFerence Detection}

When the signal of frequency is processed by the parallel device, frequency of standby generator is usually slightly higher than the online generator's, which makes the standby generator can share a little load immediately after switching on. And it can avoid reverse power[1]. Therefore, both time witch the phase change 360 degrees don't equal. In other words, the phase difference constantly changes. It is the ideal time when the phase difference is zero. Due to the operating time of the circuit breaker, core controller must to give a sign of switching on before the phase difference is zero.

Firstly, the A phase voltage of the standby generator and the online generator are transformed into a square wave signal with the same frequency by the waveform transformation circuit shown in Figure 1[2]. 


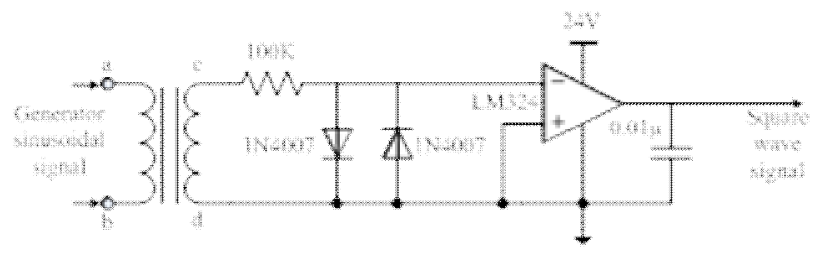

FIGURE I. WAVEFORM CONVERSION CIRCUIT.

At some point, the phase of standby generator's voltage lags online generator's $\theta$ degrees, shown in Figure 2. $T_{\mathrm{W}}$, $f_{\mathrm{W}}$ are the period and frequency of the online generator's voltage. $T_{\mathrm{F}}, f_{\mathrm{F}}$ are the period and frequency of the standby generator's voltage. $u_{\mathrm{W}}$ is the online generator's voltage. $u_{\mathrm{F}}$ is the standby generator's voltage. $\phi$ is the phase difference. For the online generator's voltage, it takes $T_{\mathrm{W}}$ to change 360 degrees, so it will take $t_{1}$ to change $\theta$ degrees.

$$
t_{1}=\frac{\theta}{360} T_{\mathrm{w}}
$$

It takes period of frequency difference $\left(T_{\mathrm{S}}\right)$ for phase difference to change 360 degrees. So it will take $t_{2}$ for standby generator's voltage to chase up online generator's.

$$
t_{2}=\frac{\theta}{360} T_{\mathrm{s}}
$$

Put (3) in (2), then (2) can be expressed in below form:

$$
t_{1}=\frac{t_{2}}{T_{\mathrm{S}}} T_{\mathrm{w}}
$$

When $t_{2}$ equals the operating time of the circuit breaker $\left(t_{\mathrm{k}}\right)$, the phase difference ( $\theta$ ) will be eliminated exactly within the operating time of the circuit breaker. Usually, $t_{\mathrm{k}}$ is known. Replace $t_{\mathrm{k}}$ with $t_{2}$, where $T_{\mathrm{s}}=\frac{1}{f_{\mathrm{F}}-f_{\mathrm{W}}}$, then (2) can be expressed in below form:

$$
t_{1}=\frac{t_{\mathrm{k}}\left(f_{\mathrm{F}}-f_{\mathrm{w}}\right)}{f_{\mathrm{w}}}
$$

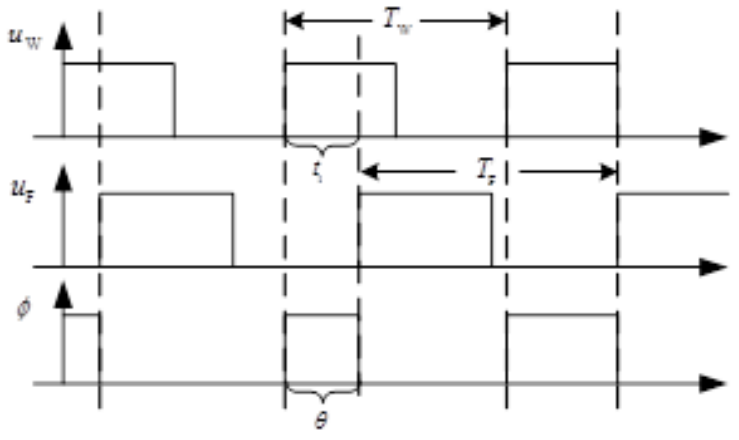

FIGURE II. WAVEFORM OF VOLTAGE AND PHASE.

It is the Constant Lead Time by the formula (5). It means: When the phase of online generator's voltage leads standby generator's voltage $t_{1}$, core controller gives a sign of switching on, which makes the phase difference be eliminated exactly within the operating time of the circuit breaker. It ensures that the phase difference is zero at the time of switching on.

Detect the lead time which the online generator's voltage leads the standby generator's all the time. When it equals to the Constant Lead Time, give a sign of switching on.

\section{Realization of Phase Difference Detection}

\section{A. Connection of Hardware Circuit}

In this paper, the phase difference detection device needs to convert the sine wave voltage signal of the standby generator and the online generator to the square wave signal with the same frequency, which needs to be accessed to the input terminal of PLC. The square wave voltage signal of online generator access to I0.1, the square wave voltage signal of standby generator access to I0.1. High speed pulse is generated by pulse width modulation, which is integrated by PLC. PWM1 is enabled in this scenario, the default output is Q0.0. For high speed counters, HSC1 is enabled, the default input is I0.0. So connect Q0.0 and I0.0. In addition, S7-1200 is CPU1214C $\mathrm{DC} / \mathrm{DC} / \mathrm{DC}$, so its power supply is DC $24 \mathrm{~V}$. The hardware circuit connection diagram of the device is shown in Figure 3, in which the principle of the waveform converter is shown in figure 1 .

In the process of the hardware connection, it is necessary to pay attention to the ground terminal of the online generator's voltage square wave converter and the standby generator's, which must be connected with the ground terminal of the PLC. Otherwise it will be affected by electromagnetic interference which can result in triggering interrupt routine inaccurately.

\section{B. Measurement of Lead Time}

According to the principle of phase difference detection, it is necessary to obtain lead time which the online generator's voltage leads the standby generator's voltage. In S7-1200PLC, the high speed counter's parameters, for example, the direction, the new initial value and the new reference value can be changed through the instruction "CTRL_HSC" in the process of program execution at any time[3]. Due to PLC with the 
method of circular scanning, it may affect the timeliness of execution, if the parameters are changed in the main program. However, the interrupts occur at a much lower rate than the counting rate of the HSC, precise control of high-speed operations can be implemented with relatively minor impact to the scan cycle of the CPU[4]. The method of interruption allows each load of a new preset to be performed in a separate interrupt routine for easy state control. Figure 4 is a schematic diagram of using the interruption mode to obtain the lead time.

In diagrams, at time $t_{0}$, the positive edge of the online generator's voltage square wave signal triggers one interrupt routine, in which the new initial value is reset as zero by instruction "CTRL_HSC". So the high speed counter is cleared, and starts to count from zero. At time $t_{1}$, the positive edge of the standby generator's voltage square wave signal triggers the other interrupt routine, in which the value of HSC is read. The result that this value is multiplied by the pulse frequency equals to the lead time. The moment which HSC is reset and read is captured by the interruption mode. So the lead time is real-time and accurate.

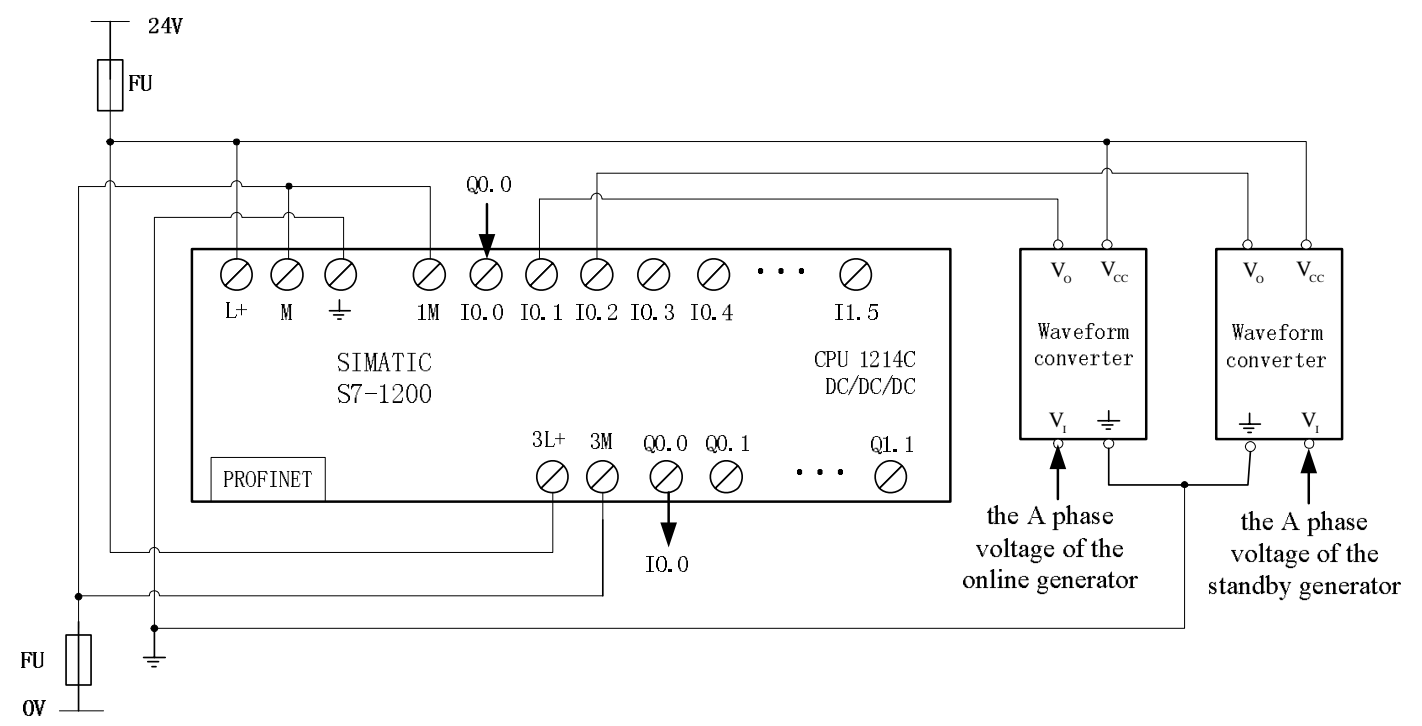

FIGURE III. THE HARDWARE CIRCUIT CONNECTION DIAGRAM OF THE DEVICE.

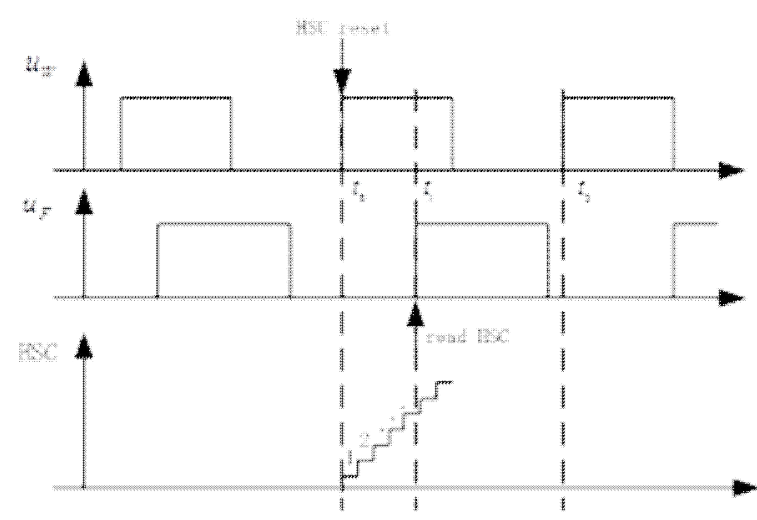

FIGURE IV. OPERATION BY INTERRUPTION MODE

\section{PLC Configuration}

In this paper, the configuration and programming of PLC are completed in Potal. To configure the operational parameters for the modules, select the module in the Device view and use the "Properties" tab of the inspector window.

Configuration of PWM1: Enable the PTO1/PWM1 (check box). Pulse generator is used as PWM. Chose "Output source" as onboard CPU output. The cycle time is set to 50 microseconds. "Initial pulse width" is $50 \%$.

Configuration of HSC1: The high-speed counters must be configured in the project settings PLC device configuration. First, enable the HSC1 (check box). Chose "Type of counting", as counting. "Operating phase" is single phase. "Counting direction is specified by" user program. Chose "Initial counting direction" as count up.

Setting of digital inputs: The input channel of HSC1 is I0.0, which is used to detect the $20 \mathrm{kHz}$ pulse. Refer to table 1 , the input filter should be set to 20 microsec. I0.1 and I0.2 are used to detect about $50 \mathrm{~Hz}$ square-wave signal. So their input filters should be set to 6.4 millisec. Because interrupt which are triggered by the positive edge of the online generator's voltage and the standby generator's, it is necessary to enable "the positive edge detection". And set names of interrupt events and the corresponding interrupt names.

Note: It will be better to set the input filter according to table 1. If the maximum input frequency which can be detected by the input filter is lower than its actual input frequency, it will lead that the PLC does not detect the input signal. If the maximum input frequency is far higher than its actual input frequency, glitches may affect detection results, which leads to trigger interrupt routine by mistake. 
TABLE I. "INPUT FILTER TIME" SETTINGS

\begin{tabular}{|c|c|}
\hline Input filter time & Maximum input frequency \\
\hline 10 microsec & $50 \mathrm{kHz}$ \\
\hline 12.8 microsec & $39 \mathrm{kHz}$ \\
\hline 20 microsec & $25 \mathrm{kHz}$ \\
\hline 0.05 millisec & $10 \mathrm{kHz}$ \\
\hline 0.1 millisec & $5 \mathrm{kHz}$ \\
\hline 0.2 millisec & $2.5 \mathrm{kHz}$ \\
\hline 0.4 millisec & $1.25 \mathrm{kHz}$ \\
\hline 0.8 millisec & $625 \mathrm{~Hz}$ \\
\hline 1.6 millisec & $312 \mathrm{~Hz}$ \\
\hline 3.2 millisec & $156 \mathrm{~Hz}$ \\
\hline 6.4 millisec & $78 \mathrm{~Hz}$ \\
\hline 10 millisec & $50 \mathrm{~Hz}$ \\
\hline 12.8 millisec & $39 \mathrm{~Hz}$ \\
\hline
\end{tabular}

\section{Programming}

In the main program $(\mathrm{OB} 1)$, the instruction "CTRL_PWM" is used to turn on the high speed pulse when paralleling. And use the instruction "ATTACH" to establish the connection between interrupt routine and the hardware interrupts event.

In the interrupt routine triggered by the positive edge of the online generator's voltage, use the instruction "CTRL_HSC" to change its parameter "CV" to 1 , "NEW_CV" to 0 . So the new initial value is set to 0 , HSC will start counting from 0 , when the next pulse arrives.

In the interrupt routine triggered by the positive edge of the standby generator's voltage, read the current value of HSC1 firstly. Theoretical value of constant lead time $t_{1}$ can be obtained by using the instructions to write the formula (5), and replacing $t_{\mathrm{k}}$ by the operation of the circuit breaker. Give a sign of switching on, when the lead time by measured equals the Constant Lead Time.

Note: CPU stores the current value of each HSC in the process image area, for example, the default process image area address of HSC1 is ID1000. The value in the process image area does not change during a scanning cycle due to the influence of the cyclic scanning mode. However, $20 \mathrm{kHz}$ pulse must make the current value of HSC1 change during a scanning cycle. So the current value must be obtained by reading peripheral address[5]. For example, the peripherals address of HSC1 is ID1000: P.

\section{E. Result of the Experiment}

Install a current transformer at the output of the online generator. Use the current probe $(100 \mathrm{mV} / \mathrm{A})$ of the oscilloscope to measure the current signal of the secondary side of the current transformer. Capture the current of the online generator at the moment of closing. The maximum, minimum and root mean square values of the current, at the process, are collected, as shown in Figure 5, Figure 6.

Figure 5 shows the change of the current under the control of FAS-113DG which is designed by DEIF. After clothing, the maximum amplitude of the current is 6.32A. The current amplitude is $2.16 \mathrm{~A}$ before closing. The biggest impulse current accounts for $192.6 \%\left(\frac{6.32-2.16}{2.16} \times 100 \%\right)$ of the current before closing. Under the same load, Figure 6 shows the change of the current under the control of the phase difference detection device which is designed in this paper. After clothing, the maximum amplitude of the current is $4.40 \mathrm{~A}$. The biggest impulse current accounts for $103.7 \%\left(\frac{4.40-2.16}{2.16} \times 100 \%\right)$ of the current before closing. By contrast, it is found that the impulse current under the control of phase difference detection device is smaller than the impulse current under FAS-113DG. It shows that the phase difference detection device which is designed in this paper is more accurate to capture the moment of closing.

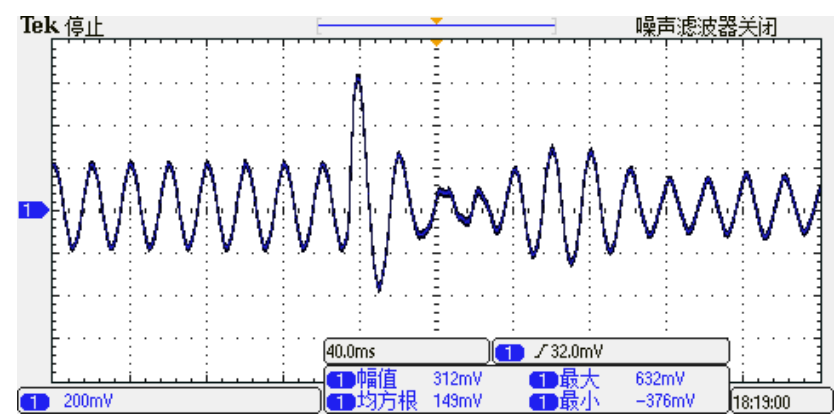

FIGURE V. IMPULSE CURRENT OF DEIF

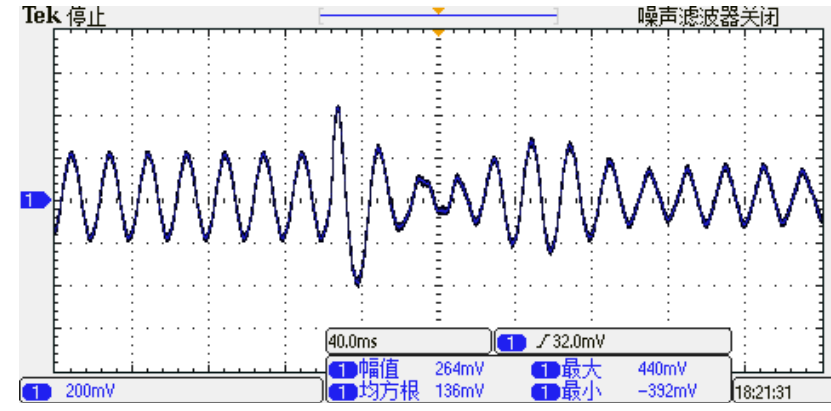

FIGURE VI. IMPULSE CURRENT OF THE CONTROLLER IN THIS PAPER.

\section{CONCLUSION}

The phase difference detection device which is designed in this paper has been tested repeatedly on the physical simulation experimental platform of marine power station. The experimental results show that the design can greatly improve the accuracy of phase difference detection. It is a reference for 
the further research and development of marine power automatic equipment.

\section{REFERENCES}

[1] Zhiliang Wu. Marine power station. Dalian: Dalian Maritime University Press, 2012

[2] Zhao Xu, Zhiliang Wu. Design of Automatic Parallel Operation Set for Marine Power Station Based on S7-1200 PLC. Marine Electric, 2014,34(11):48-51.

[3] Changchu Liao. S7-1200 PLC programming and applications. Beijing: China Machine Press, 2010.

[4] Siemens AG.S7-1200 system manual, 2009

[5] Chun Zhang. Explain profound theories of Siemens S7-1200 PLC in simple terms. Beijing: Beijing University of Aeronautics and Astronautics Press, 2009. 